\title{
Algunas consideraciones sobre el Derecho Maritimo en el Perú
}

\author{
Por el Dr. W. BACHICH DE RECINA. \\ de las Univensidades de Zagreb y Lima (P. N. C.)
}

Para estudiar la utilidad de una reforma eventual de la legislación maritima debemos apreciar brevemente la naturaleza del derecho marítimo y su lugar en el derecho en general, fijar sus fines y llegar de tal manera a esbozar algunas ideas básicas que deberán aplicarse en la legislación futura en el caso de que ésta se renueve.

Habrá igualmente que analizar el derecho marítimo vigente y sus deficiencias, las obvias a primera vista y las que resulten de un estudio mós profundo, confrontándolo también con algunas otras legislaciones, en cuanto parezcan interesantes, y estudiar las distintas instituciones del derecho marítimo.

A ráí de tal estudio preliminar se podró llevar a cabo un plan general de eventuales reformas, basadas en principios fundamentales, Y para las varias instituciones en un estudio detallado de cada una de ellas, para que resulte al fin un sistema armónico y no un simple conjunto de normas, sin cohesión y sin plarn general e ideas directrices. Aquí no se trataría de reformar por el gusto de renovar, ni por cumplir con las exigencias de uno u otro grupo interesado, sino de reformar el derecho marítimo nacional, en cuanto parezca útil para que sirva al adelanto de la economía marítima del país y de su marina mercante, para facilitar el tráfico internacional y para conseguir un adelanto mós en el desarrollo del Perú.

Habró, por ejemplo, que armonizar los intereses de los interesados directamente, de los armadores y de los tripulantes nacionales, con los intereses del país y de su economía. Habrá que tomar en cuenta que el derecho marítimo tiene sus tradiciones, quizás más que otras ramas del do recho, estrechamente ligadas con el derecho internacional y que una reforma eventual puede: ya sea alejar demasiado el derecho marítimo nacional de los principios generales, aplicados por tradición $y$ costumbres en el mundo marítimo, ya sea, al contrario, hacer que se mantengan, de manera exagerada $y$ en perjuicio del pais, principios anticuados, solamente por razón de tradiciones hoy en día ya inútiles. Visto que el derecho marí- 
timo crea o mantiene normas para regular actividades en un ambiente muy especial y complicado, quizás más variado que en otras ramas, resultará, en caso de una reforma, un trabajo de coordinación de muchos elementos que debe además apoyarse en los principios del derecho marítimo internacional, aceptados por todas las naciones marítimas del mundo.

En un breve trabajo no se puede sino esbozar todo lo necesario y la preparación efectiva de una reforma exigirá estudios mucho más detallados, pero igualmente de un trabajo reducido puede resultar la utilidad de una reforma y quizás parcialmente el rumbo que habría que tomar para aplicarla.

\section{El Derecho Marítimo Internacional.}

El tráfico entre los distintos países y continentes por vía marítima, la conexión más importante en las comunicaciones del mundo, tiene hoy en día el significado de una gran industria mundial, de un sistema único que obedece a costumbres, reglas y leyes, ya en gran parte unificadas. Estas costumbres $y$ leyes forman la mayor parte del derecho marítimo internacional o mundial y al mismo tiempo una parte considerable del derecho marítimo nacional de los respectivos países. Si tal sistema carece todavía, en muchas de sus partes, de una legislación unificada, que la impone, no sólo de hecho, sino igualmente como una legislación única, reconocida también formalmente por todo el mundo marítimo, eso es debido al hecho sensible, que el mundo estuvo hasta la fecha demasiado dividido para poder llegar a la codificación formal de reglas y leyes que de hecho ya reconoce. Pero, a pesar de esa falta de una codificación formal, el mundo marítimo llegó a universalizar una parte considerable del derecho marítimo común $a$ todos, simplemente por tradición y por necesidad.

Considerando el desarrollo histórico del complejo de tales normas, hay que mencionar los méritos de los códigos franceses en los siglos pasados y especialmente del Código de Comercio napoleónico del año 1806. copiado por muchas otras naciones en sus partes esenciales, y de las normas tradicionales norte-europeas, casi iguales a las normas francesas, que estaban vigentes en Inglaterra, en el Mar del Norte, en Escandinavia y en el Báltico, ambos sistemas, que han contribuído bastante a la unificación del derecho marítimo y al desarrollo del derecho marítimo internacional contemporáneo. Como precursora importante del derecho marítimo internacional, se debe mencionar igualmente la conferencia de Montevideo de 1888 y otro mérito importante se debe reconocer a los trabajos de las organizaciones científicas jurídicas internacionales $y \propto$ los distintos tratadistas, por su influencia sobre la codificación del derecho marítimo nacional de los respectivos países y la aplicación de las normas del derecho marítimo internacional.

Pero la mayor importancia en la unificación del derecho marítimo, en la práctica y en la vida marítima, la tiene la antigua tradición que se desarrolló por las mismas necesidades de la navegación y del tráfico entre los puertos desde tiempos muy antiguos $\mathrm{y}$ en vastas regiones, conecta- 
das únicamente por la navegación. De manera que hasta varios fragmentos de reglas o leyes marítimas de la antigüedad, que conocemos, y las reglas medioevales del Consulado del Mar, los Roles de Olerón y el Guión del Mar no representan nada más que el reconocimiento de un estado de cosas existente, el fin de un desarrollo en la respectiva época y no un inicio del derecho marítimo. Lo mismo se puede decir más o menos de las legislaciones francesas y de las normas tradicionales norte-europeas y mientrcs que en tiempos anteriores los numerosos soberanos o las ciudades independientes no hicieror mucho más que permitir, por la mera necesidad de gozar de las ventajas del tráfico marítimo, que se aplique en sus puertos y buques el derecho marítimo reconocido en amplias regiones, los grándes estados que se de sarrollaron en los primeros siglos de la edad modema, hicieron poco para la codificación del derecho marítimo internacional.

Bastará decir que el comienzo de la codificación universal se hizo esperar nada menos que hasta el año 1910, a pesar de tantos trabajos cientí ficos $y$ leyes nacionales que lograron ya mucho antes la unificación de hecho, no tanto por la voluntad de los legisladores sino por la tradición y necesidad. El mencionado primer paso de codificación universal concreta $Y$ exacta, aceptado por todos en 1910 estaba representado por las famosas reglas para evitar colisiones en la navegación, las reglas de tráfico del mar. Existía un antecedente de éstas, en una "recoriendación" franco-britómica, de hace unios 100 años, la misma que fué entonces aplicada por todos, pero cuya codificación definitiva en el mundo se efectuó solo en 1910. No cabe duda que esta codificación era una necesidad urgente para conseguir la más elemental seguridad de la navegación, porque realmente no bastaba que tales reglas sean aplicadas sólo de hecho o por la unificación de los reglamentos respectivos en los distintos países.

Al mismo tiempo comenzó otro desarrollo de gran importancia. en la codificación internacional del derecho marítimo por las llamadas conferencias diplomáticas de Bruselas, iniciadas en 1906, que dieron lugar a las CONVENCIONES DE BRUSELAAS, aceptadas y ratificadas por la mayoría de los países marítimos igualmente como derecho nacional: las dos primeras convenciones del año 1910, en materia de abordaje y salvataje; las dos del año 1924 sobre limitación de la responsabilidad de los armadores y sobre "conocimientos"; una del año 1926 sobre los privilegios y las hipotecas marítinas y las tres del año 1952 sobre la competencia civil en materia de abordaje y otros eventos del mar, sobre la competencia penal para lo mismo y respecto al embargo preventivo de buques; en su total 9 convenciones. Hay que observar que el Perú no las ratificó. Por dichas convenciones se dió un buen paso adelante, porque las mismas codificaron por lo menos una parte del derecho marítimo internacional. En el mismo campo de unificación hay que señalar igualmente las Reglas de York y Amberes sobre averías, cuya última formulación, del año 1950, abarca la respectiva materia en 22 reglas, en lugar de las 23 de la formulación amterior del año 1924. En fin, hay que mencionar las diferentes normas técnicas, universalmente reconocidas $y$ codificadas, ratificadas por casi todos los países, inclusivo el Perú, es decir las del Convenio para la seguridad de 
la vida humana en el mar del año 1929, que sin duda será pronto modernizada; las del convenio sobre líneas de flotación con las reglas para la determinación de la línea de carga máxima y de límites de las zonas y de las regiones periódicas; además las de la convención sanitaria internacional. Igualmente se puede mencionar la Convención de Bruselas del año 1938 sobre asistencia y salvataje de naves aéreas y por naves aéreas en el mar.

No obstante la mencionada codificación parcial, hay todavía mucho camino que recorrer para lograr la unificación de todas las partes del derecho marítimo que debe tener valor para todas las naciones marítimas en forma de un Código Mundial de las normas jurídicas.

El interés que tienen todos los países, por lo tanto igualmente el Perú, en la aplicación de este derecho internacional se puede al fin ilustrar por un ejemplo simple, que, en tal o semejante forma, se puede producir y se produce en la vida cotidiana marítima. Supongamos un envío de mercadería de Lisboa al Callao, por ejemplo, de una cantidad de, corcho. Como no hay líneas directas, podrá suceder que tal carga sea embarcada en Lisboa en un buque italiano que la lleve hasta Nueva York; que en este puerto sea trasbordado a un buque chileno, de la línea Nueva York-Valparaíso, que la descargará en el Callao. Tal mercadería se encontrará, por lo tanto, durante su viaje, bajo la competencia de cinco legislaciones. Si surge un conflicto, resultará inmediatamente la utilidad de que tales leyes sean iguales, como lo son de hecho para la mayoría de las materias del derecho marítimo. Pero bastará que una u otra de las naciones, cuyas leyes valdrán en tal caso, no hayan ratificado las convenciones de Bruselas, para provocar una situación que hará la solución de tal conflicto dífícil o imposible.

Aquí hay que mencionar igualmente, al lado de la unificación ya parcialmente conseguida por las convenciones de Bruselas y por las que prescriben las medidas técnicas de la seguridad de la vida humana en el mar, las normas aceptadas por la mayoría de los países para el trabajo marítimo en los últimos decenios, sea en forma de convenciones, sea como recomendaciones. Así tenemos: las 2 convenciones y 3 recomendaciones del año 1920, las 2 convenciones del 1921, las 2 convenciones y 3 recomendaciones del 1926, las 2 convenciones y 2 recomendaciones del 1929, 4 convenciones y 1 recomendación del 1936, 6 convenciones y 4 recomendaciones del 1946, 7 resoluciones breves del 1946 y las 3 convenciones del 1949, trabajo que sigue, todo preparado, concluído o por lo menos recomendado por las respectivas conferencias generales de la Organización Internacional de Trabajo en Ginebra (en 1946 en Seattle), que estón creando paular tinamente un derecho mundial de trabajo marítimo, mejor dicho, la respectiva parte del derecho marítimo intemacional. Se trata de un desarrollo, porque en varios casos las convenciones posteriores modifican partes de las anteriores o las amplían; pero, sin embargo, hay aquí un adelanto notable en la unificación y codificación de esta rama. Como existe bastante intercambio de personal entre las distintas marinas mercantes y tratándose en líneas generales del mismo ambiente, será sin duda útil para todos, si 
se llegara a una unificación general, pues actualmente, por lo menos en cuanto a los salarios, nos hallamos frente a problemas difíciles por las diferentes situaciones en cuanto al costo de la vida $y$ al valor del dinero nacional en los diversos países.

Aunque el derecho internacional marítimo exista solo en parte en normas ya codificadas, en parte en normas que, aunque no lo estén, se aplican sin embargo en todas las marinas mercantes $y$ en parte en reglas o costumbres que todavía no son aplicadas por todos, se deberá en una reforma eventual del derecho marítimo nacional tomar en consideración de manera esencial lo que el derecho internacional marítimo hoy en día considera como normas generalmente vigentes $y$ que deberían por lo tanto encontrar su expresión igualmente en la legislación nacional.

\section{Lo que significa el Derecho Marítimo para el Perú.}

Aunque en una reforma eventual de la legislación marítima nacional se deberá, por lo dicho, adaptar y aplicar las normas internacionales $\alpha-$ ceptadas en el mundo, hay otras partes del derecho marítimo, en las cuales se deberá tomar en cuenta el significado que el derecho marítimo público, lo mismo que el privado, tendrá para el país, debiendo servir al adelanto de los intereses nacionales como lo consiguen, en otras ramas, otras legislaciones especiales muy útiles, tales como el Código de Minería, la Ley del Petróleo, etc. Este significado de la legislación maritima para el Perú se podrá medir, como en otros países, según la importancia que tienen los intereses marítimos para la nación.

Examinaremos, por tanto, brevemente el derecho marítimo nacional vigente, que se compone en primer lugar del libro tercero del Código de Comercio, llamado "del comercio marítimo", título que no es exacto, porque no se trata solamente del comercio marítimo, sino de normas más amplias sobre los buques y la vida marítima. Pero, además del Código de Comercio y al lado del mismo, existe el "Reglamento de Capitanías y de la Marina Mercante Nacional". Este contiene junto con una serie de detalles de importancia secundaria y del verdadero Reglamento de Capitanías de puerto, ampliados por varias prohibiciones administrativas que llevan anexas las respectivas multas, todo un reglamento para la marina mercante y algunas normas, que deberían mejor insertarse en el mismo Código $\mathrm{Ma}$ rítimo.

Además, pertenece a la legislación marítima nacional la ley de $\mathrm{Hi}$ poteca Naval del año 1916, ley bastante moderna, que tendría lógicamente que formar parte del Código que trate de los buques en general; además la ley No. 6207 del año 1928 sobre cabotaje, cuyo contenido tendría que ser insertado en las normas generales del derecho marítimo público $\mathrm{y}$ por fin varias leyes menores $\mathrm{y}$ convenciones ratificadas, que tienen fuerza de ley y que fueron publicadas como anexos al Reglamento de Capitanías. Hasta el Código Civil contiene una norma que pertenece al derecho marítimo cuando, en su artículo 812 , inciso 49 , dice que las naves son inmuebles, lo gue no puede tener otro significado que la aplicación de 
las prescripciones del artículo 813 a los buques, sobre la definición de las partes integrantes del inmueble, porque la inscripción de los buques en el Registro especial y la hipoteca naval son previstos por normas, que no se encuentran en el Código Civil. Aquí podemos añadir que el buque podría ser definido como bien inmueble sui generis, pero igualmente como bien mueble sui generis. La verdad es, que desde los tiempos más remotos el buque es considerado en el derecho marítimo en una categoría muy espe cial, al mismo tiempo objeto de derechos $\mathrm{y}$, cuando tiene su dotación en navegación o en los puertos, como una especie de individualidad especial y en ciertos casos como una espacie de persona jurídica. Por lo tanto, no se puede simplemente definirlo como inmueble, quizás, excepción hecha, para un buque en construción o para un buque desarmado.

La posición que tiene la legislación marítima en el Perú es, por lo tanto, como en muchos países, la que se originó como consecuencia de un descrrollo no concluido, desenvuelto según las oportunidades, con ampliacicnes y cambios, aplicados según los intereses del país y así como se presentaron en los últimos 50 años. No tenemos razón alguna de criticarla, porque correspondía a su época y era tan buena como las legislaciones marítimas de otros países. Particularmente para el Libro $3^{\circ}$ del Código de Comercio hay que reconocer que ha tomado bien en cuenta el sist tema ya. unificado del derecho marítimo internacional de su época y las tradiciones internacionales, aunque actualmente parezca ya algo anticuado.

Para fijarse en el significado que tiene el derecho marítimo para el Perú, habrá que agregar algunas observaciones, porque podría presentarse hasta entre los juristas una opinión que podría ser más o menos la siguiente: ¿q́ué importuncia grande tiene pues el derecho marítimo para el Perú con su marina mercante relativomente pequeña, frente $\alpha$ los otros problemas jurídi$\cos$ y económicos nacionales, no tratándose en fin de nada más que de una parte menor, la última del Código de Comercio que prescribe las respectivas normas? Tal opinión, que suponemos, sea probablemente exagerada, puede sin embargo, existir, quizás en forma menos radical. Suprimiendo esto contestemos con una frase breve, que trataremos de probar $y$ fundamentar: EL DERECHO MARITIMO Y, CON EL MISMO, LA POLITICA MARITIMA - ECONOMICA TIENEN UNA IMPORTANCIA TRASCENDENTAL PARA EL PERU COMO PFIS MARITIMO Y DE SUं CALIDAD PUEDE MUCHO DEPENDER EL DESARROLLO ECONOMICO DEL PAIS.

Para probar tol afirmación, será útil observar que para cualquier nación vale en este sentido, que el derecho marítimo debe no solo contener normas jurídicas precisas, sino que debe ayudar por su contenido $y$ fomentar el desarrollo marítimo nacional, creando al mismo tiempo los órganos necesarios para abrirle el camino. Solo un sistema único de legislación marítima, forjado según los intereses nacionales -los cuales representan un complejo de problemas complicados pero un corjunto interdependiente- puede dar un buen resultado. No se trata solo de intereses fiscales, sino del adelanto de la economía nacional $y$ por tanto no puede 
solucionarse en una forma definitiva el problema de la legislación marítma por una serie de leyes $y$ reglamentos, editados ad hoc y por varias ramas de la cadministración pública sin coherencia suticiente.

El ejemplo de otros países puede esclarecer mejor el problema $y$ actualmente hay en el mundo varios ejemplos de legislaciones marítimas modernas, que tomaron en cuenta las necesidades $Y$ la importancia del problema marítimo para su país. Pero nos parece útil escoger a Suiza, aunque no sea $\alpha$ primera vista un país marítimo $\mathrm{y}$ aunque hace unos decenios se acostumbraba todavía hacer chistes sobre la "marina suiza", porque este país no tiene ni costa ni puertos, excepción hecha de los puertos fluviales del Rhin y hasta hace poco tiempo no tenía ni marinos. Suiza se encuentra, sin embargo, hoy en el camino ya muy adélantado de crear y fomentar su nueva marina mercante, que corresponde $\alpha$ los intereses de este país en cuanto transporto por el mar una parte de la mercadería que sale de Suiza o llega a esta, pasando por los puertos del Medi terróneo, del Mar del Norte o del Atlántico, o bien después o antes de un trasbordo en los buques fluviales suizos del Rhin. La nueva marina mercante suiza porticipa igualmente en el tráfico internacional, asegurando así las respectivas ganancias al capital suizo y a las tripulaciones de sus buques, que paulatinamente se constituyen y completan con suizos, que se dedican $\alpha$ esta profesión nueva para los habitantes de la Confederación Helvética. Ya en el año 1941 dictó Suiza su primera y buena ley marítima que por razones constitucionales tuvo entonces el carácter de una ley provisional; además organizó una Dirección para la Marina Mercante en su Gobierno Federal. Ya que la ley provisional debía ser reemplazada por una definitiva, haciéndola votar por su Casa-de Representantes, el Gobierno presentó a esta una nueva ley, algo modificada y ampliada, 'যn Có digo Marítimo, que abarca todos los respectivos problemas, que fué ampliamente discutido por la Casa de Representantes y por el público y entonces aprobada (1) y que tiene el fin de hacer progresar los "intereses marítimos suizos". Citaremos algunas palabras de la declaración introductiva del proyecto de dicha ley del diputado del Ständerat suizo Dr. Schoch, que dijo: "nuestro comité considera como natural $y$ evidente que la navegación marítima suiza, que se desarrolló de manera favorable durante los últimos 12 años $y$ que se implantó bien en el mundo, debe ser conservada y que debe dársele un ordenamiento jurídico definitivo. Tomando en consideración el abastecimiento de nuestro país, sería irresponsable abandonar lo que ya conseguimos. Sin embargo, también, sin relación con este problema, la navegación marítima representa para nuestro país económicamente un gran activo, por lo cual tenemos todas las razones de cuidarlo bien".

En cuanto a los detalles del contenido del Código marítimo suizo hay que mencionar que sus primeros 10 artículos comprenden, siguiendo la ley dal año 1941, los principios generales, la orgamización de la autoridad.

(1).-Aprobada el 23 de setiembre de 1953, promulgada el 20 de febrero de 1956, en v1. gencia desde el $1^{\circ}$ de enero de 1957. 
administrativa central del Gobierno suizo para la marina mercante y la jurisdicción de los juzgados para la misma. Podemos agregar que el título segundo trata de los buques suizos, de su inscripción, de los derechos reales sobre los buques y de los documentos de los buques. El tercer título trata de la dirección técnica, del armador y del capitán; el cuarto de las tripulaciones con normas generales, contratos de servicio y seguridad social. El quinto título se ocupa de los contratos marítimos; el sexto de eventos y accidentes, el séptimo de la aplicación del derecho marítimo en la navegación interna -fluvial y lacustre- y el octavo representa la parte penal con delitos contra la seguridad de la navegación, contra el orden y la disciplina y "contra la navegación suiza" $y$ al fin sigue un reglamen. to de penas disciplinarias. Las convenciones de Bruselas de 1926 sobre reglas comunes de hipotecas marítimas y las de 1910 sobre colisiones de buques y salvataje. Además las reglas de York y Amberes en su formulación del año 1950 aparecen como anexos al Código, pero con fuerza de ley (2).

No cabe duda que la marina mercante suiza se desarrolló ya bajo el régimen de la ley del año 1941 de una momera rápida, que su desarrollo sigue $\mathrm{y}$ que pasó ya en 1953/54 el límite de una marina modesta, cuando llegó a tener 40 buques con 150,000 toneladas bruto $Y$ unas 200,000 toneladas de portada, con un valor de cerca de 10 millones de dólares, adelanto que sigue enteramente por iniciativa paricular y capital privado.

Hemos citado este ejemplo de Suiza, porque confirma en un caso particularmente interesante, lo que la legislación debe significar para la actividad $y$ el progreso marítimo de una nación, sirviendo a fortalecer las bases para un desarrollo sano de la marina mercante y de la parte marítima de la economía nacional. Mucho depende en cada país de la "Política Marítima" y económica de su gobierno y la legislación debe ser su resultado, pero al mismo tiempo también su instrumento y su fúndamento sólido. Se desprende de lo dicho el segundo elemento importante que desempeña su papel en una reforma eventual del derecho marítimo, es decir que este debe servir al adelanto de la marina mercante y de los intereses económicos del país, armonizándolos con los intereses sociales. Si eso vale para cualquier país marítimo, hasta para Suiza, eso valdrá también para el Perú, que, como otros países, tiene su marina mercante, una antigua tradición marítima y su economía marítima, la misma que representa el desarrollo de su economía nacional en los mares. No hay razón alguna, para que tal actividad de un país debiera cesar o ser muy reducida más allá de sus espigones o de los lanchones que cargan los buques. No hay ni razón para que un país no tome parte -y parte importante- en el tráfico marítimo alimentado por su economía nacional. Nadie piensa hoy en día monopolizar para su propia bandera el tráfico de sus puertos, pero todos, hasta Suiza, tratan de asegurarse una parto adecuada de su propio tráfico. Eso lo podrá conseguir todo país por las

(2).-Suiza ratificó en 1954, todas las convenciones de Bruselas. 
medidas respectivas, aunque indirectamente y no por imposición directa. Vamos a citar un ejemplo. Sabemos muy bien que, por varios acuerdos de colaboración entre los armadores extranjeros, conferencias, etc., los buques peruanos quedaron excluídos del servicio de líneas que unen a los puertos peruanos con los norteamericanos y que por el momento no será fácil que la bandera nacional tome parte en las líneas con Europa. Sea lo que fuera, actualmente, siempre hablando de líneas más o menos regulares, unas 15 ó 20, que tocan mensualmente los puertos peruanos y que sirven para abastecer una gran parte del comercio exterior de importación del Perú, y para exportar una parte de los productos que produce el país, son todas de bandera norteamericana, inglesa, francesa, italiana, alemana, holandesa, danesa, sueca, noruega y chilena; pero ninguna de bandera nacional. Esos buques izan por cortesía y costumbre la bandera peruana al entrar en nuestros puertos, en su palo de trinquete, pero no tienen tripulantes peruanos $y$ de sus ganancias nada le queda al Perú. No es cuestión de exagerar y de pretender que una parte fuerte diel servicio de líneas con Europa y Estados Unidos sea servida por buques pleruanos, pero será resultado siempre deseable de la futura política marítima nacional eliminar los elementos que lo impidan y conseguir, de cualquier manera, que una parte adecuada del tráfico marítimo se realice bajo la bandera peruana y que sea servida por la iniciativa y actividad indus. trial nacional, sola o en colaboración con otros, así como ya lo consiguieron para sus marinas, Chile, Brasil y Argentina. Eso se puede conseguir lenta y sistemáticamente, como todo el desarrollo marítimo nacional, pero uno de los elementos para lograrlo será una legislación marítima adecuada, que lo ayude.

Confrontando el Perú como país marítimo con otras naciones para las cuales vale todo lo explicado, debemos destacar que lo dicho debería valer mucho más para el Perú que para la mayoría de los otros países. Porque el Perú se debe considerar desde el punto de vista del tráfico marítimo, como una isla. No menos que en los países-islas el tráfico y el comercio internacional, el que une al Perú con el extranjero, pasa únicamente por la vía marítima, porque no existen otras posibilidades. El tráfico de frontera de Tacna, Puno y Tumbes cuenta poquísimo y la región de la Selva, on cuanto conectada directamente con el extranjero, lo es también por la vía del Amazonas que pertenece al tráfico marítimo. Fuera de la vía del mar $y$ del tráfico local de frontera, el Perú está conectado con el extranjero sólo por la vía aérea, lo mismo que una isla. Hasta hace pocos años se hizo también la conexión interna en el país para regiones importantes y para una gran parte de los productos por el mar. Eso sigue todavía para varios de estos productos pero un muchos casos la vía marítima ha sido reemplazada por el camión, en perjuicio de la navegación de cabotaje. Pero todavía hay que probar si esta situación actual no es debida a las dificultades que encuentra la navegación de cabotaje $y$ en primer lugar el embarque y la descarga en los puertos. Sin embargo sigue la navegación de cabotaje como factor muy importante en la vida económica nacional y si, hasta que no se organice la red ferroviaria con la Selva, el Oriente Perua- 
no ha sido poco conectado con el centro occidental del país, eso se debe a una deficiente conexión de esta rama muy especial del cabotaje nacional, cabotaje en el sentido jurídico, que es el servicio de navegación Iquitos. Amazonas-Ponamá-Costa Peruama, lo que precisamente se trata de remediar en la actualidad.

El Perú es, por lo tanto, en el fondo, más que otros poíses, una nación que depende del mar, una nación marítima por su naturaleza, aunque no se ha adaptado todavía completamente a esta situación; ni siquiera ha adaptado su economía a ella, no fomentando bastante lo que podríumos llamar la prolongación o continuación de la economía nacional en el mar. Erar natural que, cuando el descrrollo industrial y comercial no había todavía salido cie su estado de iniciación, no se pudo pretender que la marina mercante se desarrolle mucho y sin duda tenía ella su vida adecuada a las condiciones de entonces. Quizás era la marina mercante en períodos pasados relativamente más importante que ahora y no hay que olvidar las entonces modernas líneas rápidas nacionales de cabotaje, que existían hace deco nios por mérito de la Compañía Nacional de Vapores. Tenemos una institución marítima relativamente pequeña que por sus buques $Y$ su servicio puede servir de ejemplo, aunque se dedica sólo $\alpha$ su servicio y queda parcialmente inutilizada durante pate del año, es decir la flota guanera. Tenemos además, la Corporación de Vapores de dimensiones impor. tantísimas en su tonelaje, con una actividad siempre mayor en el transporte de productos de masa $\mathrm{y}$ con una participación en la navegación libre a veces importante, la misma que mantiene también una parte del servicio de cabotaje, pero la iniciativa privada falta en el momento actual casi completamente para llegar a un verdadero progreso de la marina mercante que corresponda a los éxitos conseguidos en otros campos industriales $y$ económicos.

Teniendo en cuenta la importancia de la vida marítima para el $\mathrm{Pa}$ rú vemos que resalta igualmente la importancia del derecho marítimo en nuestros conceptcs. Ahora bien, se puede observar que únicamente la política marítima, la iniciativa $y$ no el derecho marítimo pueden contribuir al adelanto del país en el sentido marítimo. A tal observación se puede contestar que el derecho marítimo, además de darle a la vida marítima una base ordenada, deke abarcar también todas las medidas administrativas, todo lo que constituye "el ordenamiento jurídico marítimo", como lo llamó el diputado suizo que hemos mencionado. $Y$ bien logrado tal ordenamiento $Y$ creada la unidad gubernamental, que lo aplique eso se materializará indirectamente en el progreso de la industria marítima nacional de la misma manera como sucede en otros países y como lo demuesíra el ejemplo de Suiza, donde 7 empresas de iniciativa absolutamente privada, están interesadas en la marina mercante. Con el desenvolvimiento mayor de la industria del armamento nacional se conseguirá el adelanto de todos los que viven o podrían vivir de la actividad marítima, que el número de estos aumenten, $Y$ que lo que se debe forzosamente gastar en tramsportes marítimos, alimenfados por el Perú y su economía, sea en gran parte, útil a la vida nacional, a su capital y a su trabajo. Lo que resulta de esta tercera parte del tema 
expuesto es, como hay que repetirlo, que el ORDENAMIENTO JURIDICO MARITIMO NACIONAL debe ser tal que pueda hacer progresar y garantizar. el desenvolvimiento de la economía marítima nacional en la mejor forma posible, armonizando los intereses de todos los elementos participantes.

Además, habrá que fomentar la navegación de cabotaje que, confrontada con la importancia relativa que tenía en otras épocas de la historia nacional ha sido, sin duda, reducida. No sabemos, si los propietarios de los kinques de cabotaje y los marinos interesados en ellos por su ocupación, lo han hecho ya presente a los sectores competentes; pero no cabe duda que mientras el Estado está indirectamente subvencionando el tráfico de camiones no facilita el tráfico marítimo de cabotaje. Si el Estado proporciona $-\mathrm{y}$ hace bien en hacerlo- medios al tráfico terrestre, construyendo carreteras y poniéndolas sin pago alguno a disposición del tráfico de carros de cualquier tipo, el mismo Estado hace poca cos $\alpha$ para el cabotaje y no pone ni los puertos a disposición gratuita del mismo. Aquí no se irata de pedir que se dificulte el tráfico terrestre sino de destacar la necesidad de facilitar en todo lo posible el tráfico de cabotaje marítimo. Se puede uno preguntar qué cosa tiene este problema que ver con la legislación marítima, pero la contestación es lo que ya dijimos, que solo una legislación marítima adecuada puede unificar todos los esfuerzos del Estado para darle a la marina mercante en general y al cabotaje en particular las facilidades que garantizarán su futuro desarrollo. $\mathrm{Si}$ un Ministerio construye los puertos, otro se ocupa de la policía marítima y ninguno de la política marítima económica, no puede haber tales esfuerzos, o por lo menos no estarán bien dirigidos y coordinados. Una ley fundamental, que regule la competencia de la autoridad central coordinadora para la marina mercante y que imponga a todas las autoridades, por el contenido de sus normas, las ideas directrices, será, por lo tanto, una condición esencial de un buen desarrollo.

Una política marítima bien dirigida y basada en una buena ley tendría muchos medios para ayudar a la marina mercante nacional y particularmente al cabotaje.

Varias cuestiones relacionadas con una reforma eventual del Derecho Maritimo Nacional.

Aparte de los principios fundamentales habrk que estudiar -con respecto a una reforma eventual- todas las instituciones del derecho marítimo, una por una, para ver como habrá que cambiarlas o adaptarlas: primero, en cuanto difieran del derecho internacionalmente adaptado, por ejemplo, en materia de las convenciones de Bruselas, abordaje, salvataje, conocimientos y responsabilidades de los armadores. Aquí puede ser útil el método de insertar partes en la nueva Codificación y añadir otras como suplemento a la ley en su forma de convención ratificada con fuerza de ley, como lo hace el Reglamento de Capitanías de 1950 para varias convenciones ratificadas por el Perú y como lo hace el nuevo código suizo para cuatro de las nueve convenciones de Bruselas. Segundo, habrá que 
ver, cuales normas se deben, ya sea reformar o insertar como nuevas para regular, facilitar y fomentar la vida marítima.

Para redactar los detalles se debe hacer estudios exactos de cada parte con las respectivas exposiciones de motivo, llegando así a un texto más o menos reformado, modificado o ampliado. El marco de esta trabajo no permite entrar en ciertos detalles y por tanto se esbozarán sólo algunos comentarios generales o particulares que posiblemente puedan ser útiles en el estudio preliminar.

10 ¿Hay que seguir a no con el sistema de insertar la legislación marítima en el Código de Comercio? Aunque sea como "libro" particular. como lo hizo el Código de Comercio de 1902.

El argumento a favor del sistema existente es en primer lugar un argumento de tradición $y$ esto no sólo de tradición peruana, sino de tantos otros países y especialmente de la ley comercial napoleónica, que se aplicó en muchos países. Además, se dice que se trata de normas comerciales, siendo la actividad marítima sólo una parte del comercio, en cuanto a los contratos, carga, conocimiento, seguro, etc. Pero hay igualmente fuertes argumentos para una ley o leyes especiales. Considerando todo el Ordenamiento Jurídico marítimo como una unidad, abarcando el derecho público y administrativo marítimo y el social, todo este conjunto de normas no cabe en el Código de Comercio, pero por otro lado tendrá grandes ventajas, como ya dijimos, de forjar toda la legislación marítima dentro de un sistema único. Como todo eso no cabe en el Código de Comercio, será por tanto mejor sacar la respectiva parte de este Código e insertarla como una de las partes de un nuevo "Código Marítimo". Aunque parezca que parte de las normas tengan un carácter comercial, se puedie, igualmente, destacar que estas normas serón de una naturaleza bastante particular, que serán relaciones sui géneris, características para el ambiente marítimo. Los tratadistas tienen la tendencia de confrontar las distintás instituciones $\mathrm{y}$ así se aplica a cada institución del derecho marítimo definiciones y comparaciones con otra institución correspondiente del derecho en general, especialmente del derecho civil, del derecho que podríamos llamar "derecho terrestre" en relación con el marítimo, aunque habrá juristas que considerarán esta definición un "horribile dictum". Porque, por ejemplo, encapricharse en la definición del buque del cual ya hablamos, comenzando por tratarlo de mueble $\mathrm{y}$, como no alcanza tal defirición, a causa de la hipoteca naval, de ir al otro extremo, llamándolo inmueble, cuando el buque es absolutamente un concepto sui géneris, como objeto muerto una "res mari adscripta", un objeto que no puede alejarse del mar y como objeto vivo, es decir, un buque que funciona, con su capitán, con su tripulación, casi una especie de individuo responsable y además una persona jurídica sui generis. No se puede negar que un buque solicita permisos, envía mensajes, se carga de responsabilidad, tiene su sello y contrae deudas. Si provoca daños, se responsabiliza el buque y en segunda línea la persona responsable que lo dirige. Puede suceder que dos buques que pertenecen al mismo propietario tengan un conflicto entre sí, por ejemplo en caso de colisión o salvataje; lo que no puede producirse en otras ra. 
mas del derecho, fuera del derecho aéreo, que es algo así como un derocho marítimo aplicado a otro ambiente físico. Así que el mismo buque, ca mo concepto casi fundamental del derecho marítimo en la mayoría de sus partes, es un elemento inmanente de casi todas las instituciones: por tener un buque en su propiedad el armador es armador; por no solo conducir, sino manejar en todos los sentidos su buque, el capitón es capitán, y lo mismo vale en menor grado para los distintos miembros de la tripulación y para los pasajeros, que son tales, mientras se encuentron en el buque.

Los contratos valen para los buques $y$ el delito marítimo o la infracción de la disciplina marítima no existen si no son cometidos a bordo o en relación con un buque. Y hablando de salvataje y colisión, las normas modernas fijan en primer lugar la responsabilidad de cada buque como tal. Todo esto lo mencionamos para subrayar la naturaleza bastante particular del derecho marítimo, la que existe desde tiempos inmemoriales y para probar que, si puede ser constatada una relación con el comercio en general, tal relación comercial no es más que un aspecto de las relaciones marítimas, cuyos otros aspectos son de naturaleza diferente. Recordémosnos de los eventos que pueden suceder en el campo del derecho marítimo y eso no excepcionalmente, sino con cierta frecuencia $\dot{y}$ como eventos, que tal derecho abarca, para comprender más fácilmente que se trata de una rama muy diferente. Supongamos las horas o los días durante los cuales un buque estó luchando con el mal tiempo o la actividad de un buque en este caso, cuando busca a otro, para salvarlo, o la maniobra para tal salvataje y para no ir tan lejos hay que recordar los frecuentes eventos tan críticos en el mar, para comprender que la navegación es una cosa muy especial y no una parte del comercio. No hay razón, por tanto, para que no existiere en todos los países un código marítimo, como lo tienen ya muchas naciones y por lo menos una colección de leyes, dos o tres, que en su conjunto formen la legislación marítima, como en otros países, pero que sea un sistema único y con disposiciones bien relacionadas entre sí.

Aquí hay que mencionar otro argumento más para la redacción de un Código marítimo independiente. La nueva redacción de un Código de Comercio vendrá con el tiempo, pero exigirá mucho trabajo. Si la legisłación marítima debe igualmente en el futuro formar parte del Código de Comercio, se perderá más tiempö. La reforma del Código de Comercio y del derecho marítimo por separado podrá hacerse con más rapidez, si se prepara cada uno independientemente. La tendencia modema de crear Códigos y leyes especiales, como lo aplica el Perú ya con éxito en varias nuevas leyes (Minería, Petróleo, Electricidad, etc.), para cada rama de la vida nacional, impone a fortiori la creación de un código especial para una rama tan importante como la marina mercante nacional.

29 Habrá que prever una parte general del Código marítimo o do la legislación maritima por la cual se fijarám algunas normas fưndamentales, que pueden ser completadas eventualmente con varias reglas de derecho marítimo público sobre jurisdicción, puertos -on este último caso teniendo en cuenta la convención-estatuto de los puertos de 1923. generalmente aceptada como teoría. Tal parte puede contener deta- 
lles de un carácter muy delicado, como, en cuanto a la libertad del mar y la jurisdicción nacional, materia que debe ser estudiada desde muchos puntos de vista y que ya ha sido bien tratada en el Perú.

39 El Código deberá prever que autoridades administrativas y judiciales serán competentes para la aplicación de la legislación marítima, porque sin tales cuerpos que vigilen con atención y estrictamente su aplicación y dejando eso a la competencia de varias organizaciones, que tie nen también otras competencias $\mathrm{y}$ cuya responsabilidad por tanto no será 'bien establecida, se llegará a la misma desorganización a la cual llegaría, por ejemplo, la minería sin la existencia de una Dirección de Minas. Varios países, entre ellos algunos que dependen menos del inar que el Perú, tienen su Ministerio de la Marina Mercante, otros ur Secretariado de Estado, algunos una dirección ministerial, pero muy raro es el caso, donde no existe una organización administrativa en el seno del gobiemo, especializada $y$ dedicada únicamente a la marina mercante y a le: economía marítima del país. Dividida tal competencia ente varios ministerios o diferentes organizaciones, no se puede llegar a un buen éxito. Además necesitará un Consejo Superior Marítimo, sea con voto consultivo, sea únicamente para armonizar los intereses de los distintos elementos. Si no fuere el Código el que prevea todo eso, así como el Código de Minería prevé la respectiva organización, será difícil que una organización correspondiente tenga una vida estable, la única que garantiza resultados que podrón en este campo conseguirse sólo con años de trabajo sistemático. Pero no basta la autoridad administrativa en la esfera del poder ejecutivo. Aquí se necesita también una autoridad judicial especial, que podría componerse de uno o más juzgados privativos especiales, relacionacins eventualmente con "consejos de expertos". Podrían serlo también unos juzgados comunes en uno o varios puertos, con asesores expertos, siempre con la eplicación de varias normas particulares de procedimiento.

4․ Una parte importantísima de la cual puede depender esencialmente el adelanto de la marina mercante de una nación es la que en el libro 3 del Código de Comercio actucl abarca en la sección "de los buques" cuya makeria está en parte integrada $y$ en parte reglamentada por el "Preglamento de Capitanías y de la Marina Mercante Nacional", segunda parte, títulos VI y VII: Marina mercante y Material de la marina mercante. Sin entrar en detalles, se trata de facilitar a la iniciativa particular: la participación del capital en la marina mercante, la actividad de los armadores, la creación de empresas navieras, la compra y la construcción de buques. Lo facilita sin duda la norma contenida igualmente en leyes de otros países y que prevé el Código de Comercio en el libro tres art. 602, que dos o más personas, partícipes ien la propiedad de un buque, se presumen formar una compañía de copropietarios, pero lo dificultan muchas otras normas, como la llamada "fianza de bandera". Se necesita sin duda un control, $y$ se deben impedir abusos, pero tal efecto se consigue mejor con normas penales, sin imponer una fianza que alcanza una fuerte parte del valor del buque. Aunque no será oportuno quie los propietarios sean extranjeros, no será desfavorable que haya participación extranjera, la 
cual por ahora no se permite formalmente. El registro debe ser rápida y el procedimiento simplificado y no se debe olvidar, que hay países que ayudan a su marina mercante con subvenciones o primas, para la construcción de un nuevo buque; con primas para viajes realizados y por medio de subvenciones para líneas que funcionen. Hay, además, ejemplos, que capital extranjero se invierte en buques mercantes de otras banderas, no en el sen. tido de lo que podemos llamar "buques sin nacionalidad", los que tienen una "bandera ficticia" (como, por ejemplo, la de Libería), pero de manera adecuada y con grandes ventajas para el país, en cuya marina se inviertan, lo que se debería facilitar mediante la legislación, siempre cuidando los intereses nacionales.

5 . En cuanto $\alpha$ las normas sociales y las que se refieren al servicio del personal habrá que adaptar cuanto más las convenciones internacionales y garantizar al personal según el espíritu contemporáneo los derechos correspondientes, creando buenas condiciones de trabajo, pero garantizando también un buen trabajo. Habria que considerar también, si todas las normas sociales deberían ser insertadas en el mismo código o en otra ley particular $\mathrm{y}$ en reglamentos.

69 Habrá que insertar también las normas de la hipoteca naval en el Código marítimo y habrá que eliminar el préstamo a la gruesá como anticuado.

Habrá, en cuạnto se quiere o debe reformar la legislación marítima, que redactar un nuevo Código, que signifique un paso adelante para la marina, los marinos, la economía nacional y por tanto para el Perú. E trabajo será complejo, pero el resultado valdrá la pena. 